Supporting Information for

\title{
Low-Dimensional Carbon and Titania Nanotube Composites via a Solution Chemical Process and Their Nanostructural and Electrical Properties for Electrochemical Devices
}

\author{
Sunghun Eom ${ }^{1}$, Sung Hun Cho ${ }^{1}$, Tomoyo Goto ${ }^{1}$, Myoung Pyo Chun ${ }^{2}$, and Tohru Sekino ${ }^{1} \dagger$ \\ ${ }^{1}$ The Institute of Scientific and Industrial Research (ISIR), Osaka University, 8-1 Mihogaoka, \\ Ibaraki, Osaka 567-0047, Japan \\ ${ }^{2}$ Korea Institute of Ceramic Engineering and Technology (KICET), Soho 101, Jinju, \\ Gyeongsangnam 52851, Korea \\ †sekino@sanken.osaka-u.ac.jp
}

\section{Experimental}

\section{Chemical reagents:}

The following chemical reagents were used for the present study. Multi-wall carbon nanotube (MWCNT, outer diameter $=10-20 \mathrm{~nm}$, inner diameter $=5-10 \mathrm{~nm}$, length $=0.5-200 \mu \mathrm{m}$, purity $>95 \%$, Sigma-Aldrich Japan, Tokyo), Graphite (Particle size $<45 \mu \mathrm{m}$, Wako Pure Chemical Co., Tokyo, Japan), $\mathrm{H}_{2} \mathrm{SO}_{4}$ (Sulfuric acid, 95\%, Wako Pure Chemical Co., Tokyo, Japan), $\mathrm{KMnO}_{4}$ (Potassium permanganate, 99\%+, Sigma-Aldrich Japan, Tokyo, Japan), $\mathrm{H}_{2} \mathrm{O}_{2}$ (Hydrogen peroxide, 30\%, Wako Pure Chemical Co., Tokyo, Japan)), Titania (P25, $\mathrm{TiO}_{2}$, Degussa, Germany), $\mathrm{NaOH}$ (Sodium Hydroxide, 97\%, Wako Pure Chemical Co., Tokyo, Japan).

Electrical resistivity measurement for the powder samples:

The electrical resistivity of the prepared powder samples was measured by the Van der Pauw method at room temperature using specially designed jig (see Fig. S1). The jig was made from 
hard and transparent plastic (acrylic resign) to hold a powder inside of it (7 $\mathrm{mm}$ in diameter). The powder thickness is approximately $0.2 \mathrm{~mm}$ in the jig. Four platinum (Pt) wire electrodes are put into the powder through the upper disk-type cover.

The measurement was carried out under the controlled constant current mode with $0.1 \mathrm{~mA}$ at room temperature, where the stability of measured electrical resistivity was sufficiently high, and the standard noise of each measurement was below 3.0\%.

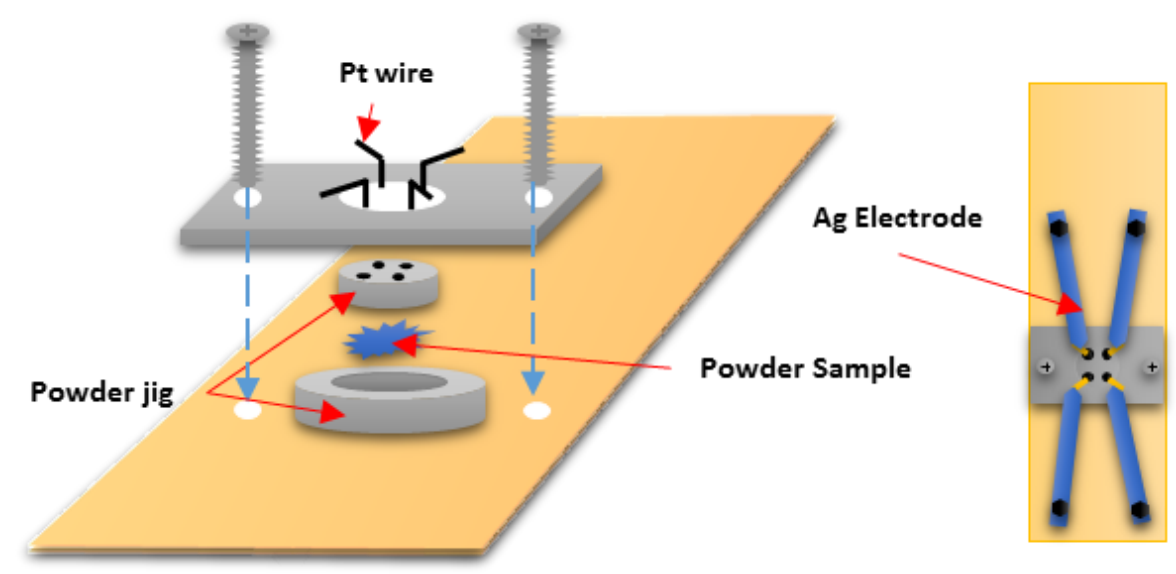

Figure S1. Schematic illustration of the cell configuration used for the electrical resistivity measurement for powder samples by the Van der Pauw method (four-point probe measurement).

\section{Results}

Fourier transform infrared spectroscopy (FT-IR):

FT-IR spectra for the pure TNT, CT, and GT nanocomposites were measured using FT/IR 4100 spectrometer (JASCO Corp. Tokyo, Japan) installed in the Comprehensive Analysis Center, ISIR, Osaka University, by diffused reflectance method. Powders were mixed with $\mathrm{KBr}$ powder and then pressed to disks. The results were shown in Fig. S2. 


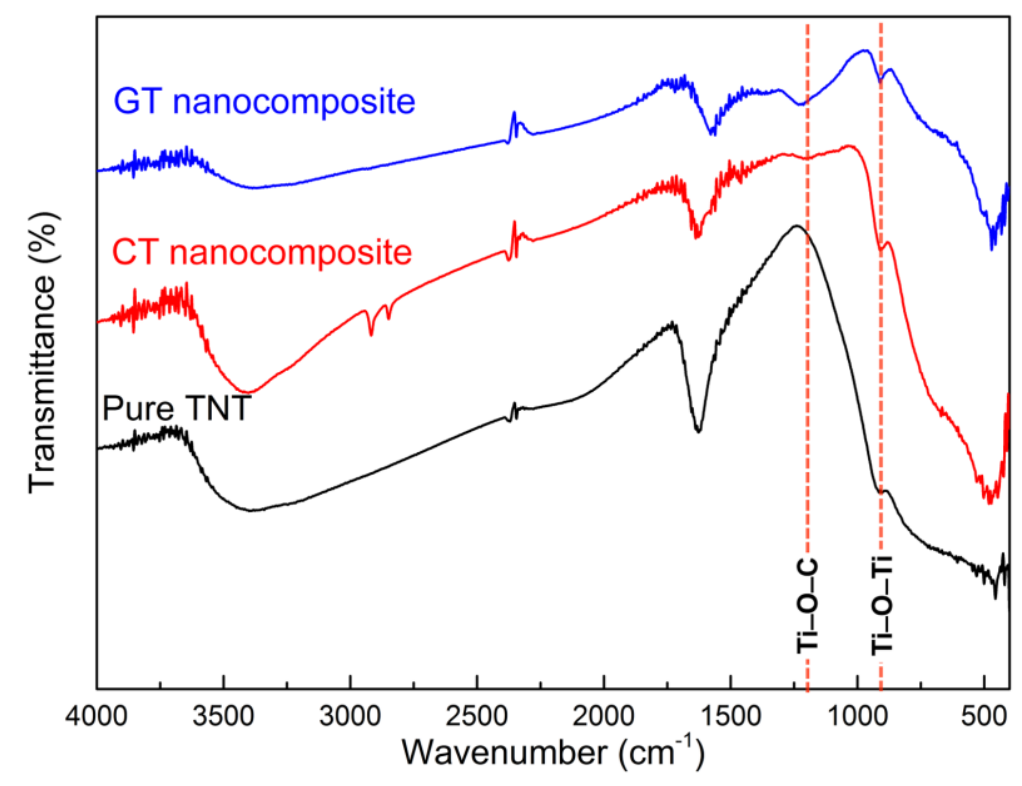

Figure S2. FT-IR spectra for the pure TNT, CT, and GT nanocomposites.

\section{Morphological characteristics:}

The both CT and GT nanocomposites have unique morphology. The schematic illustrations of the two low-dimensional carbon/titania nanotube hybrids are shown in Fig. S3, where the contact area between carbon and oxide are largely different due to their hybrid structures.

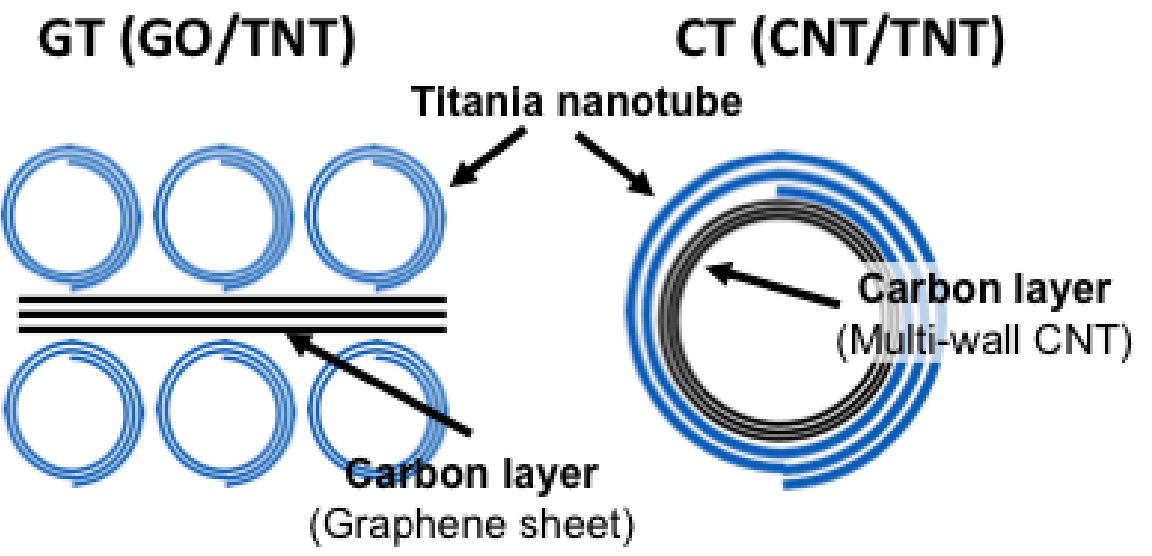

Figure S3. Schematic illustrations of the hybrid morphologies for GT (GO/TNT) and CT (CNT/TNT) nanocomposites. 